\title{
Research and Design of an Indoor Wireless Location System Based on a SOC Location Engine
}

\author{
Jian Zhao ${ }^{1, a}$, Chang Zhang ${ }^{2, b}$ \\ ${ }^{1}$ Zhengzhou Institute of Information Science and technology, china \\ ${ }^{2}$ Zhengzhou Institute of Information Science and technology, china \\ azhaojian_zz@126.com, bzcmail123@yahoo.com.cn
}

Keywords: Indoor Location RSSI Location Engine Network Construction

\begin{abstract}
According to the needs of the practical application in the indoor environment by the wireless localization method with high precision and low cost, an indoor location system based on the SOC location engine is designed, using ZigBee network and RSSI information, we also give the system scheme with the reference node and blind nodes to construct the location network, layout structure and the work flow are also presented and finally realize it. The system can location the blind node precisely with a little cost to meet the needs of indoor wireless localization.
\end{abstract}

\section{Introduction}

According to the application environment, the existing wireless location technology can be divided into two categories: one is the outdoor location technology within a wide range, such as GPS, Beidou, TOA, TDOA, AOA location, AOA / TOA mixed and so on, another is the indoor location technology within a short distance, such as optical tracking, A2GPS, combination of radio wave and ultrasonic, radio frequency identification technology (RFID) and RSSI etc [1,2,3,4,5].

Presently the domestic and foreign researches on location system based on wireless sensor network mostly calculate the blind node position through software, which acquire parameters required for their own position from the blind node, transmitted to the master control node, then acquire the coordination of the blind node through a series of complex calculation, and then transferred it to the blind node $[6,7,8]$. Because of the large amount of computation, it is necessary to use PC or high performance microprocessor, calculations required for network traffic will be increased exponentially with increase of the number of nodes, high traffic load and insufficient bandwidth makes the algorithm is not suitable for the construction of low power consumption of the wireless location system, especially for a network of battery powered, it's only suitable for a small-scale network.

With the SOC of CC2431 which launched by TI Company with a location engine embedded in it, we can use it to acquire the location of a node in the wireless sensor network without adding any other infrastructures. The scheme can acquire a high precision and a little cost, so it is very suitable for indoor location service [9]. An indoor wireless location system is presented in this paper based on the location engine of the SOC.

\section{Design of the System Scheme}

The System Scheme of the indoor wireless location is shown in Figure 1, including a wireless location network and terminal equipment for system control.

Wireless location network is rational layout according to the actual application environment, using the ZigBee wireless network module, the topology of the location network can be chain or mesh, and the network contains some blind nodes, reference nodes and a coordinator node, where the coordinator node will receive the location information from the blind node and transmit to the control terminal. 


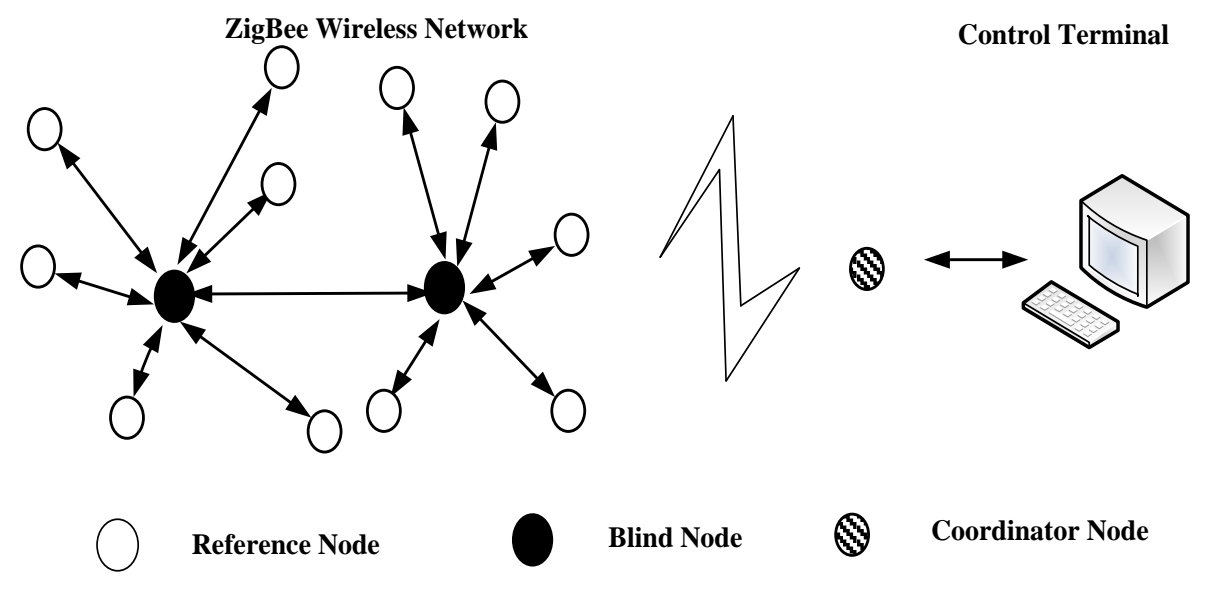

Fig1 positioning system structure diagram

The reference node is a static node located in a known position, which knows its own position and can transmit its location information to other nodes by data packets. The blind nodes receive the data packet from the reference nodes, obtain the reference node location coordinates and the corresponding RSSI value.

The blind node is structured based on the CC2431 with a location engine in it. The blind node issues a location request to the reference nodes and receives the response data packets which contain a reference node coordinate $(\mathrm{x}, \mathrm{y})$ information from the reference node, and then read out the received RSSI value, the value is sent to meditation engine. We can read out the location information calculated by the location engine and send it to the control terminal equipment. In the location network each blind node can execute the location operation, so blind node only transmits the location information calculated by the location engine, not the data used to calculate the location, which greatly reduced the amount of the data transmitted in the network.

The control terminal is in order to facilitate the user to obtain the location information, and interact with the user by the terminal equipment. Typical control terminal is a general-purpose computer, palm computer or mobile phone, which connects to the wireless sensor network by the coordinator node, so that the user control terminal can exchange information with the ZigBee network, in order to obtain information about the location of the blind node. The control terminal can store and display the location information from the wireless network.

\section{Location Engine}

The location engine of CC2431 is based on RSSI location technology. As the received signal strength indicator (RSSI) location, it's known that the transmitting node's signal strength, and the receiving node calculate the propagation loss of the signal according to the received signal strength, the transmission loss is transformed into distance using the theoretical and empirical models, and then calculate the node location use some existing algorithms[9].

The theoretical value of RSSI can be expressed by the following formula:

$$
\text { RSSI }=-(10 n \cdot \lg d+A)
$$

Among them, $\mathrm{n}$ represents the signal propagation constant, also called spread index, which represents the reduction rate of the signal energy with the increasing of the distance, $d$ represents the distance between the transmitter and the receiver, A represents the absolute value of the received signal strength with a distance of $1 \mathrm{~m}$, and the unit is $\mathrm{dBm}$. In the omnidirectional antenna mode, such as the signal strength of $-40 \mathrm{dBm}$, the A parameter is set to 40 .

The location engine of CC2431 will calculate the location information as long as the required parameters are written to it, can then the results can be read out directly. The required parameters are listed in Table 1, and the output parameters are shown in Table 2. 
Table 1 inputs parameters

\begin{tabular}{|c|c|c|l|}
\hline name & Min. & Max & \multicolumn{1}{|c|}{ Description } \\
\hline A & 30 & 50 & $\begin{array}{l}\text { The absolute RSSI value in dBm 1 meter apart for a } \\
\text { transmitter. }\end{array}$ \\
\hline N_index & 0 & 31 & This value represent the signal propagation exponent. \\
\hline RSSI & 40 & 95 & Received Signal Strength Indicator is measured in dBm. \\
\hline $\mathrm{X}, \quad \mathrm{Y}$ & 0 & 63.75 & $\begin{array}{l}\text { These values represent the } \mathrm{X} \text { and } \mathrm{Y} \text { coordinates relative to a } \\
\text { fixed point. }\end{array}$ \\
\hline
\end{tabular}

Table 2 Location Engine Output

\begin{tabular}{|c|c|c|l|}
\hline name & Min. & Max & \multicolumn{1}{c|}{ Description } \\
\hline $\mathrm{X}, \quad \mathrm{Y}$ & 0 & 63.5 & $\begin{array}{l}\text { These values represent the calculated X and Y coordinates } \\
\text { relatively to a fixed point. }\end{array}$ \\
\hline
\end{tabular}

The location engine calculates the position of the node to be located using the RSSI measurements from the reference nodes. Because of the changes of the RSSI along with the antenna design, the environment and the other RF source nearby, in order to achieve the best location accuracy, the absorption of the ceiling or floor in the environment should be considered when place the reference node. In order to ensure that the indoor location engine performance, at least eight reference nodes in the network are needed in an office environment, every node should be in the same height, and away from the floor, ceiling and walls. When the numbers of the reference node are increased in the network, the dependence of the node's test results can be decreased, and the accuracy of the location can be improved.

Most application scenarios require the location engine has a larger coverage, It can be achieved by two methods: one is to improve the output power of the reference node while reducing the precision of the calculation results of location engine. Another is to place a large number of reference nodes in a greater range, and calculation the location only use the reference node with the strongest signal. Since the second method can expand the coverage of the location engine without sacrificing the accuracy of the location, it is preferable. The concrete working principle is that the nodes to be located in the network broadcast some information, and collect the data from the adjacent reference nodes, select the strongest reference nodes' $\mathrm{X}$ and $\mathrm{Y}$ coordinates. Then, the coordinates of the other nodes associated with the reference node are calculated. At last, the data in the location engine is processed, and the distance from the nearest reference node is considered, and the actual location of the node in the large network is obtained.

\section{Implementation of the System}

The design and implementation of indoor wireless location system based on the location engine includes two parts: hardware and software. The hardware design includes the design of the reference node, the blind node and the coordinator node, which constructed based on the TI SOC [10].

Embedded Workbench development tool IAR is used to develop the location software, and it is involved in two key technologies: (1) ZigBee node identification. Each ZigBee node has 64 permanent addresses as its unique identifier. (2) Locating determination. The accuracy of the location is determined by the density of the reference nodes.

The workflows of the location system based on the ZigBee network are shown in Figure 2. 


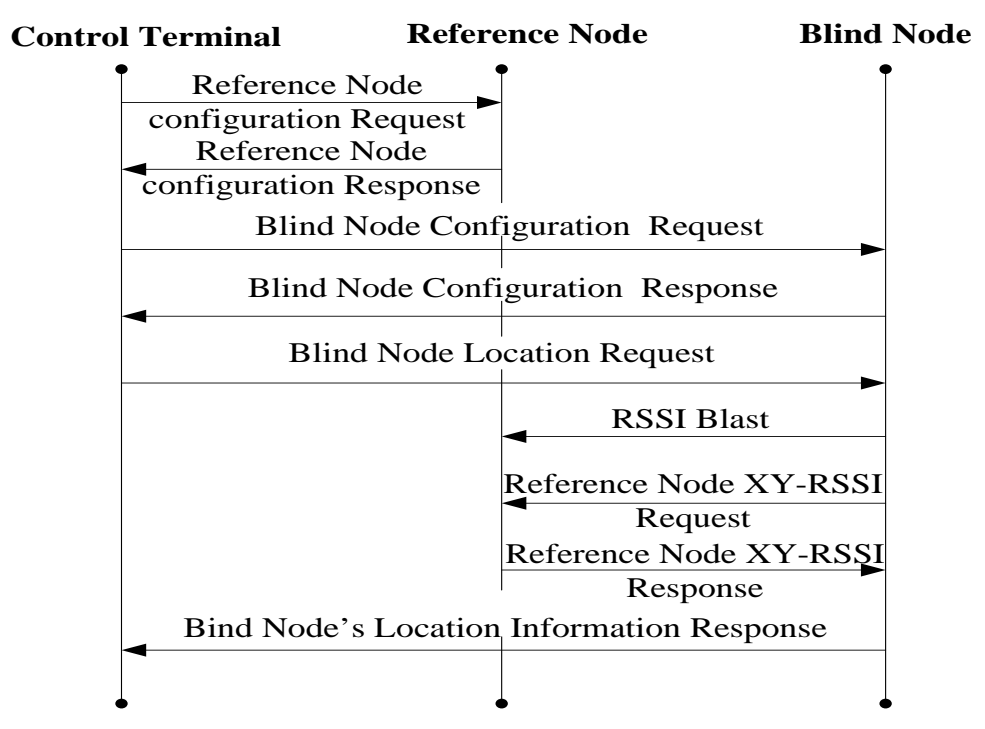

Figure 2 Location system workflow

A. The Broadcast Stage: The blind node in the ZigBee network sends a single hop broadcast information to find the reference nodes within the communication area, then sends a series of broadcast information to these reference nodes which calculate and save these packets of RSSI values.

B. Data Acquisition Stage: The blind node sends a unicast information to the reference nodes within the communication range, to query the RSSI values of the reference nodes by the successive broadcast of the 1 Stage. Reference nodes will send their location information and the RSSI value to the blind node. The blind node selects these reference nodes' data with a larger RSSI value to operation, such as setting the reference node number is 8 , the blind node woll choose these RSSI values in the first eight reference nodes, if the reference node number is less than 8 , all the reference nodes are used.

C. Calculation Stage: After received each reference node's coordinates and RSSI value, the blind node put these information to the location engine, so that the location can be calculated and send it to the coordinator node. In the design, the network traffic for location services only appeared in the blind node communication within the region of nodes and the network traffic will only increase with the the number of blind nodes. Therefore, multiple blind nodes can be located in a same network, and the system has low power consumption and low network load.

\section{Conclusions}

Compared with the software location method, the indoor wireless location system based on the SOC location engine has some advantages such as fast location, high accuracy, no occupancy of CPU time and so on. The working process of the system in the location estimation algorithm need $3 \sim 8$ reference nodes, the location estimation within $0.5 \mathrm{~m}$ as a unit, and the test results show that calculation node location takes less than $40 \mu \mathrm{s}$, ranging up to $64 \mathrm{~m}$, and location deviation are below 3 $\mathrm{m}$. In addition, the location engine is distributed computing method, useing the known reference nodes' RSSI location information, these nodes are in distributed loation so that it can effectively avoid a large number of network transmission overhead and communication delays emerged in the centralized computing methods.

\section{Reference}

[1] S.N.Gezici: submitted to Journal of Wireless Personal Communications (2008)

[2] J.H.Zhao, D.M.Wang: submitted to Journal of Engineering Sciences(2011)

[3] Y.P.Huang: diploma thesis, Nanjing University of Posts and Telecommunications (2012) 
[4] J.H.Kang: diploma thesis, Beijing Jiaotong University(2012)

[5] X.W.Shi, H.Q.Zhang and G.H.Deng: submitted to Journal of Computer Measurement and Control (2012)

[6] W.Zhang, Q.Sun: submitted to Journal of Computer Science(2014)

[7] F.B.Wang, L.Shi and F.Y.Ren: submitted to Journal of Software(2005)

[8] X.C.Sho, H.Y.Xu: submitted to Journal of Wuhan University (Engineering Edition)(2015)

[9] Information on http://www.ti.com/

[10] Y.B.Guo, K.W.Yang and J.Zhao,in: ZigBee technology and application edited by Defense Industry Publisher ,Beijing(2010) 